\title{
Refined laboratory simulations of dark-speckle coronagraphy
}

\author{
A. Boccaletti ${ }^{1}$, C. Moutou ${ }^{2}$, and L. Abe ${ }^{3}$ \\ 1 DESPA, Observatoire de Paris-Meudon, 5 Pl. J. Janssen, F-92195 Meudon, France \\ e-mail: boccalet@despa.obspm.fr \\ 2 ESO Santiago, Chile \\ e-mail: cmoutou@eso.org \\ 3 Observatoire de la Côte d'Azur, Département Fresnel, ISA-GI2T, F-06460 Saint Vallier de Thiey, France \\ e-mail: lyuabe@obs-azur.fr
}

Received June 2; accepted September 29, 1999

\begin{abstract}
Dark-speckle coronagraphy with large telescopes is expected to image extrasolar planets. We present new results about the dark-speckle coronagraphic camera, obtained in the laboratory with a simulated atmosphere and an adaptive optics bench. In good seeing conditions, and with an accurate subtraction of a reference frame, we show that our instrument allows high-resolution, high-contrast imaging, more effectively than classical long exposure, for magnitude differences larger than 5 at visible wavelength. With the present capability of the adaptive optics system used, on a $1.5 \mathrm{~m}$ telescope pupil, we should expect to detect stellar companions $10^{4}$ fainter than the primary star. Instrument concepts and processing techniques are discussed.
\end{abstract}

Key words: methods: data analysis - methods: laboratory - techniques: interferometric — binaries: close - planetary systems — brown dwarfs

\section{Introduction}

Instrumental development of adaptive optics and coronagraphic concepts are required for the improvement of image contrast (Malbet et al. 1995; Malbet 1996). Astronomical advances such as circumstellar imaging, substellar objects and exoplanet detection or the study of extragalactic nebulosities would benefit of an increased dynamic range. Limitations come from the light diffracted by the telescope and instrument optics: polishing defects, spider arms, and the wavefront residual bumpiness. The principle of dark-speckle imaging, proposed in 1995 by Labeyrie, and refined since (Boccaletti et al. 1998a)

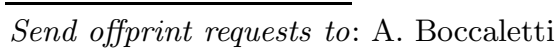

is one of the necessary steps for a better suppression of this scattered light. It requires an efficient adaptive optics and then uses the speckle imaging for freezing the atmospheric turbulence, which further degrades the image. The concept is fully described in the aforesaid papers (Labeyrie 1995; boccaletti et al. 1998a). We here present a recent experimental simulations performed with the dark-speckle coronagraph. They were obtained under good and stable artificial seeing conditions and allowed us to calibrate our instrument in comparison with the classical long-exposure imaging. We stress upon the fact that dark-speckle theory doesn't take into account optical defects of the instrument, and consequently, the results may appear optimistic in some cases. Sections 2 and 3 briefly describe the experiment and data processing. Sections 4 and 5 discuss the results.

\section{Laboratory simulations}

The laboratory tests have been carried out at the ONERA (Office National d'Études et de Recherche Aérospatiales) headquarter. A special device warms up the air confined in a tank and generates the equivalent of a single-layer turbulent atmosphere. Temperature and speed are calibrated to provide an adjustable set of values $\left(D / r_{0}, \bar{v}\right)$, where $D$ is the pupil diameter, $r_{0}$ is the Fried parameter and $\bar{v}$ is the average wind speed. The disturbed wavefront originating from an artificial light source (a binary star for example) is then restored with the BOA adaptive optics (AO) system (Conan et al. 1998). The 88 actuators of the deformable mirror allow seeing compensation at visible wavelength with a fast time response of $1 \mathrm{~ms}$. The coronagraphic camera was installed downstream the AO bench. 


\subsection{The coronagraph}

The coronagraphic instrument has already been described in detail in Boccaletti et al. (1998b). Here, additional care was taken for the design of the apodizing device, the Lyot stop, located in the relayed pupil. For a more efficient cancellation of the diffracted light, this stop has to overlap the pupil image, including its central obscuration and the supporting spider, having 3 arms at $120^{\circ}$. Three stops with different sizes and shapes were made by F. Gex and her collaborators, at the Observatoire de Paris. We have selected, with both computer simulations and laboratory tests, the most efficient stop for suppressing diffracted light. With such a Lyot stop, the dark region defined by the mask in the coronagraphic images is no longer visible and an attenuated image of the Airy peak tends to appear at the center, with an intensity similar to the speckle field (see Figs. 2, 3 and 4).

Some of the limiting factors for the Lyot coronagraph (Lyot 1939) come from inaccurate adjustment of the two main instrument components: the Lyot mask and the Lyot stop. Setting up is made even harder when working on faint objects. In order to understand some misadjustement effects such as alignment defects or longitudinal misplacement of the coronograph's elements, one of us (L.A.) has developed a numerical model of the optical set-up including not only the coronagraph itself, but also the partial correction of the AO and the detector. As explained before, the Lyot stop tends to concentrate some of the remaining light into a central peak in the coronagraphic plane. Simulations have shown that a misalignment error for the stop exceeding $15 \%$ of its diameter results in a nearly total extinction (90\%) of the central peak. Some experimental images also provided evidence of alignment drift. Further studies should allow a more accurate testing of such effects, for providing a reproducible setup of the instrument.

The coronagraphic frames are imaged on the detector with a high magnification (f/976) to achieve a fine sampling of 153 pixels per speckle area.

A Wynne device (Wynne 1979) has been included in the coronagraph to compensate the speckle chromatism as needed for dark-speckle observations with enough spectral bandwidth. The Wynne corrector, previously tested in a telescope run (Boccaletti et al. 1998b), provides a quasi-achromatic Airy pattern on a wide spectral band $(650-850 \mathrm{~nm})$.

The Lyot mask diameter is $5.16 \lambda / D$ (about 3 Airy radii) and the angular distance between the primary and the companion is $7.63 \lambda / D$.

\subsection{Data acquisition system}

The photon-counting camera CP20+ is an updated version of the CP20 (Abe et al. 1998), which was used on a previous observing run in October 1997 (Boccaletti et al. 1998b). This system is divided into three main components: the camera itself, the photon centroiding electronics and the real-time data acquisition computer.

The camera allows single photo-event detection and a very low dark count: less than 10 photons per $20 \mathrm{~ms}$ exposure, which corresponds to approximately $510^{-4}$ photon/s/pixel for $50 \mu \mathrm{m}$ pixels at $-20^{\circ} \mathrm{C}$.

Even though the centroiding electronics was limited to a reliable limit of 50000 photons/s (1000 photons per 20 ms short exposure), the low flux we were dealing with, allowed us to use the real-time facilities of the system such as live integration and display. The data files, recorded with CP20+, contain space-time coordinates of photoevents.

\section{Data reduction}

\subsection{Data processing}

A code, written in $\mathrm{C}$ language to analyse the photon coordinates delivered by the camera, generates two kinds of data set:

- the long exposure image, obtained by co-adding the frames (mapping of single photon events);

- the dark-speckle image, obtained by mapping the zerophoton events on each frame before co-adding them to generate a negative "cleaned image".

The zero-photon occurrence denotes a lack of light, and therefore the "cleaned image" is presented in negative form.

Labeyrie (1995) and Boccaletti et al. (1998a, 1998b) have explained how the dark-speckle method improves the detection of faint structures. It requires fine sampling to achieve very low light level detection with an optimal value given in Boccaletti et al. (1998a):

$j=0.62 \frac{R}{G}$

where $j$ is the number of pixel per speckle area, $R$ the star/planet brightness ratio, $G$ the star/halo brightness ratio, referred to as the adaptive optics gain by Angel (1994). The ratio $R / G$ is therefore the relevant parameter for imaging faint companions. For a companion 10 times fainter than the average speckled halo, a sampling of 6.2 pixels/speckle area is needed. The fainter the companion, the finer the sampling needed.

To adjust the sampling with respect to the ratio $R / G$, the zero-photon events counting can be made on "big" pixels containing $2 \times 2$ or $4 \times 4$ physical pixels. A zero-photon event is recorded if the photon count is strictly zero in this group of physical pixels. This process is more efficient than box averaging in this context, and thus enables to change the pixel sampling on dark-speckle images. Since the detector has near zero read-out noise, the signal to noise ratio is not degraded through this operation. 
The initial sampling on the camera was therefore set fine enough to allow such grouping of pixels. Diluting the photon rate also prevents the camera saturation. To avoid a significant loss of resolution, the groups are kept smaller than $4 \times 4$ pixels. With $1 \times 1,2 \times 2$ and $4 \times 4$ pixels/group, the sampling is then respectively $153,38.3$ and 9.5 pixels/speckle area. The latter is very close to the Shannon sampling.

The final images are still dominated by the fixed residual speckles, as mentioned in Boccaletti et al. (1998b). These static defects originate in fixed aberrations and from the residual amplitude pattern on the pupil (spider for instance). The coronagraphic images of the binary and the reference stars are recentered and scaled in intensity for subtracting this residual speckle pattern, on both the longexposure and the dark-speckle image. The map subtraction is quite efficient far from the central star but becomes less effective near the mask, where speckles can be brighter than the hidden companion.

No attempt was made to correct flat-field and darkcurrent, since they can be neglected at first order, with such a photon-counting camera.

\subsection{Data interpretation}

We describe hereafter, how estimates of the primary/secondary intensity ratio, the $\mathrm{AO}$ gain, and signal-to-noise ratios were derived.

\subsubsection{The intensity ratio}

The intensity ratio was measured on the long exposure by integrating the pixel intensities on an area $S$ encircling the $1^{\text {st }}$ bright ring of the Airy pattern, as follows:

$R=\int_{S} \frac{I_{*}\left(x-x_{0}, y-y_{0}\right)}{I_{*}\left(x-x_{\mathrm{c}}, y-y_{\mathrm{c}}\right)-I_{\mathrm{ref}}\left(x-x_{\mathrm{c}}, y-y_{\mathrm{c}}\right)} \mathrm{d} x \mathrm{~d} y$

where $I_{*}$ is the intensity distribution of the binary star with $\left(x_{0}, y_{0}\right)$ and $\left(x_{\mathrm{c}}, y_{\mathrm{c}}\right)$ the Airy peak coordinates of respectively the primary star and the companion. $I_{\text {ref }}$ is the intensity distribution of the reference star (scaled in intensity to the binary star) and describes the contribution of the star's diffracted halo at the companion location.

$I_{*}\left(x-x_{0}, y-y_{0}\right)$ is measured on off-axis image where the primary star Airy peak remains visible while $I_{*}\left(x-x_{\mathrm{c}}, y-y_{\mathrm{c}}\right)$ and $I_{\mathrm{ref}}\left(x-x_{\mathrm{c}}, y-y_{\mathrm{c}}\right)$ are measured on the on-axis coronagraphic images.

The subtraction of the PSF halo $\left(I_{\text {ref }}\right)$, measured on the reference star, is needed for accurate brightness ratio measurement, since in most cases the star halo intensity is brighter than the companion itself.

The photometric accuracy with the photon-counting camera is rather poor, owing to the imperfect photoncentroiding (Thiébaut 1994) achieved by the electronics. The photometric accuracy achieved this way is about 0.2 magnitude.

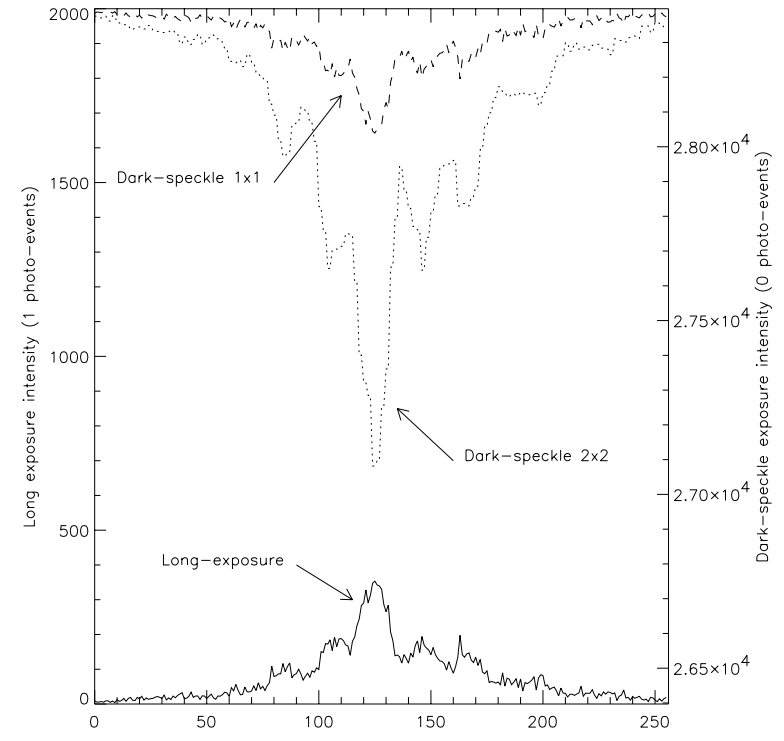

Fig. 1. Coronagraphic image profiles showing the peak (respectively the dip) of the companion at position $x=124$. Long exposure (solid line) and dark-speckle image with $1 \times 1$ pixel/group (dashed line) are stricly equivalent but displayed differently, as indicated in Sect. 3.2.3. The long exposure has positive value with 0 minima, while the dark-speckle exposure is presented in negative form with a maximum value equal to the total number of frames. The dotted line shows similar darkspeckle analysis processed with a $2 \times 2$ binning. The $S N R$ is significantly improved

\subsubsection{The AO gain}

The AO gain $(G)$ is defined by Angel (1994) as the ratio of the star's Airy peak intensity to the speckled halo intensity. $G$ is thus an estimator of the residual scattered light, and improves with the AO performance. Since the halo is smoothed on the long exposure, an accurate estimate of the average gain can be obtained at the companion's location using the expression:

$G=\int_{S^{\prime}} \frac{I_{*}\left(x-x_{0}, y-y_{0}\right)}{I_{\mathrm{ref}}\left(x-x_{\mathrm{c}}, y-y_{\mathrm{c}}\right)} \mathrm{d} x \mathrm{~d} y$

Here, the integration is performed over speckle area $\left(S^{\prime}\right)$.

With the BOA adaptive optics, under good seeing conditions and with moderate pupil diameter (see Sect. 4), it is therefore possible to reach a gain of about $100-200$ at the companion location $(7.6 \lambda / D$ from the Airy peak). A companion about 5 magnitudes fainter than the star will thus be easily detected in a long exposure on which the speckled halo is smoothed to this intensity level $(G)$. A dark-speckle analysis imaging is therefore required to improve the detection of fainter companions $(\Delta m>5)$. 

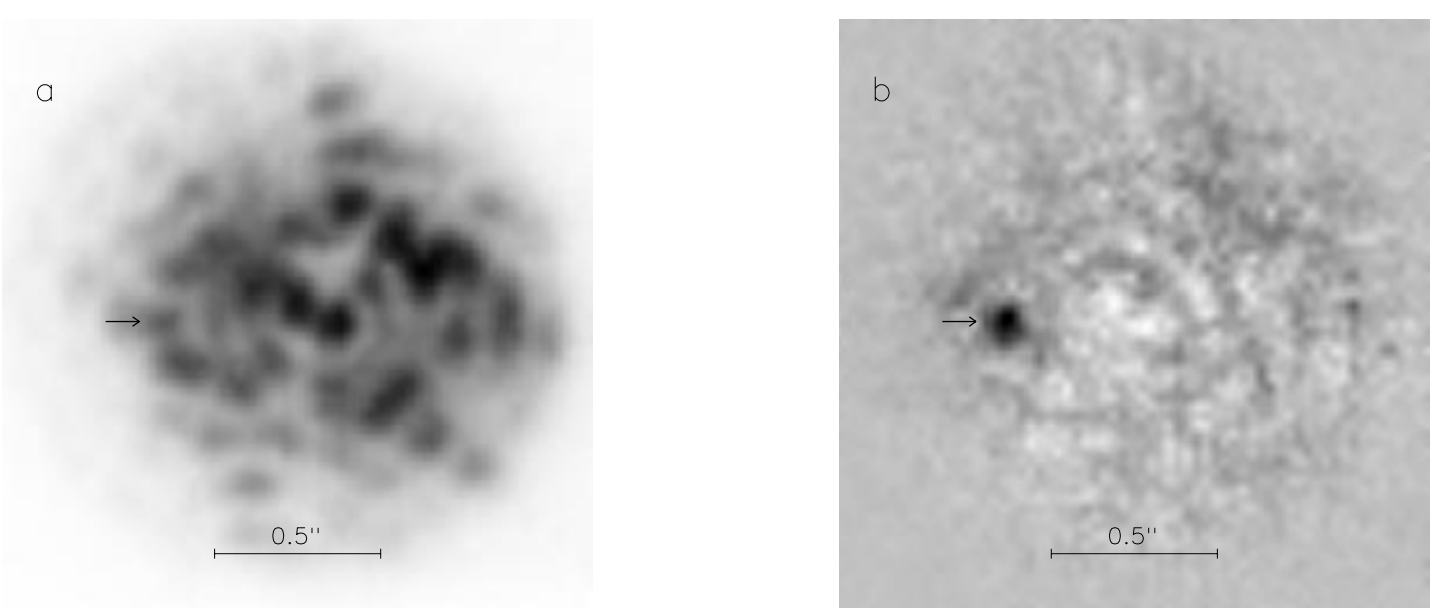

Fig. 2. Sample 1: Coronagraphic image of the binary a) and subtraction with a reference star b) for the sampling of $4 \times 4$ pixels/group, maximizing the $S N R$. The brightness ratio between the primary and the companion (arrow) is 6.4 magnitudes. Here, the subtraction is almost perfect

Table 1. Parameters measured on long exposure, (sample 1)

\begin{tabular}{lccccc}
\hline & $\Delta m$ & $G$ & $S N R_{1}$ & $S N R t_{1}$ & $S N R_{\mathrm{g}}$ \\
\hline $\begin{array}{l}\text { Long exposure } \\
1 \text { photo-event }\end{array}$ & 6.4 & 155 & 87.9 & 53.9 & 27.7 \\
\hline
\end{tabular}

Table 2. Dark-speckle $S N R$, (sample 1)

\begin{tabular}{lcccc}
\hline Sampling & $S N R_{\mathrm{ds}}$ & $S N R \mathrm{th}_{\mathrm{ds}}$ & $S N R \mathrm{sc}_{\mathrm{ds}}$ & $S N R_{\mathrm{g}}$ \\
\hline $1 \times 1$ pix/group & 6.8 & 31.7 & 78.0 & 28.9 \\
$2 \times 2$ pix/group & 30.2 & 59.4 & 175.3 & 51.7 \\
$4 \times 4$ pix/group & 107.3 & 97.4 & 314.8 & 80.8 \\
Theoritical & - & 134.6 & - & \\
\hline
\end{tabular}

\subsubsection{The signal-to-noise ratio}

Theoretical expressions of the signal-to-noise ratio $S N R$ are:

$S N R_{1}=\frac{G}{R} \sqrt{\frac{T}{t}}$

for the long exposure (Angel 1994), and:

$S N R_{\mathrm{ds}-j}=\frac{N_{*}}{R} \sqrt{\frac{t T}{j+\frac{t N_{*}}{G}}}$

for the dark-speckle exposure (Boccaletti et al. 1998a). Where $T$ is the total integration time, $t$ the short exposure time or the speckle lifetime in second unit, $j$ the sampling parameter (pixels/speckle area) and $N_{*}$ the photon rate of the star (photons/s).

Once the pixels are grouped in $1 \times 1,2 \times 2$ or $4 \times 4$ pixels on the dark-speckle exposure, 3 different $S N R_{\mathrm{ds}}$ can be evaluated.

The goal of these laboratory tests was to assess the validity of the dark-speckle model (Boccaletti et al. 1998a), and therefore only the local noise was considered to derive the $S N R$. Since it does not take into account the residual speckle noise due to static aberrations, this process leads obviously to an optimistic $S N R$, but more consistent with theoretical expressions.

At the companion location, the $S N R$ is measured over a speckle area $\left(S^{\prime}\right)$, on both the long exposure and the dark-speckle image, according to the following relation:

$S N R=\int_{S^{\prime}} \frac{\left|I_{\mathrm{ref}}\left(x-x_{\mathrm{c}}, y-y_{\mathrm{c}}\right)-I_{*}\left(x-x_{\mathrm{c}}, y-y_{\mathrm{c}}\right)\right|}{\sqrt{I_{\mathrm{ref}}\left(x-x_{\mathrm{c}}, y-y_{\mathrm{c}}\right)}} \mathrm{d} x \mathrm{~d} y$

$I_{*}\left(x-x_{\mathrm{c}}, y-y_{\mathrm{c}}\right)$ and $I_{\mathrm{ref}}\left(x-x_{\mathrm{c}}, y-y_{\mathrm{c}}\right)$ represent the 1 or 0 -photon events count respectively with and without companion.

As explained hereabove, the intensity of the companion's Airy peak on the binary star, is compared to the residual intensity at the same location on the reference star. The noise originating from static aberrations is thus not accounted in the $S N R$ calculation, and a realistic comparison with the model becomes possible.

At this point, it is possible to compare measured (Eq. 6) and expected $S N R$ (Eqs. 4 and 5), separately for the long exposure and the dark-speckle exposure. But the direct comparison of the dark-speckle and the long exposure efficiency is not straightforward. The long exposure has positive values and 0 minima while the dark-speckle image is presented in negative form with a maximum value equal to the total number of frames (Fig. 1). Therefore, dark-speckle images $\left(I_{\mathrm{ds}}\right.$ in Eq. $\left.(7)\right)$ have to be scaled to the long exposure, with the following expression:

Iscaled $_{\mathrm{ds}}=\max \left(I_{\mathrm{ds}}\right)-I_{\mathrm{ds}}$

Once $S N R_{\mathrm{ds}}$ is re-calculated on the dark-speckle scaled image, both $S N R_{\mathrm{l}}$ and $S N R_{\mathrm{ds}}$ can be accurately compared (Sect. 4). As mentioned above, the photon-counting camera provides only the 0 and 1-photon events. The long exposure $S N R_{1}$ and the dark-speckle $S N R_{\mathrm{ds}-1}$, obtained with the finest sampling, are therefore strictly equivalent 

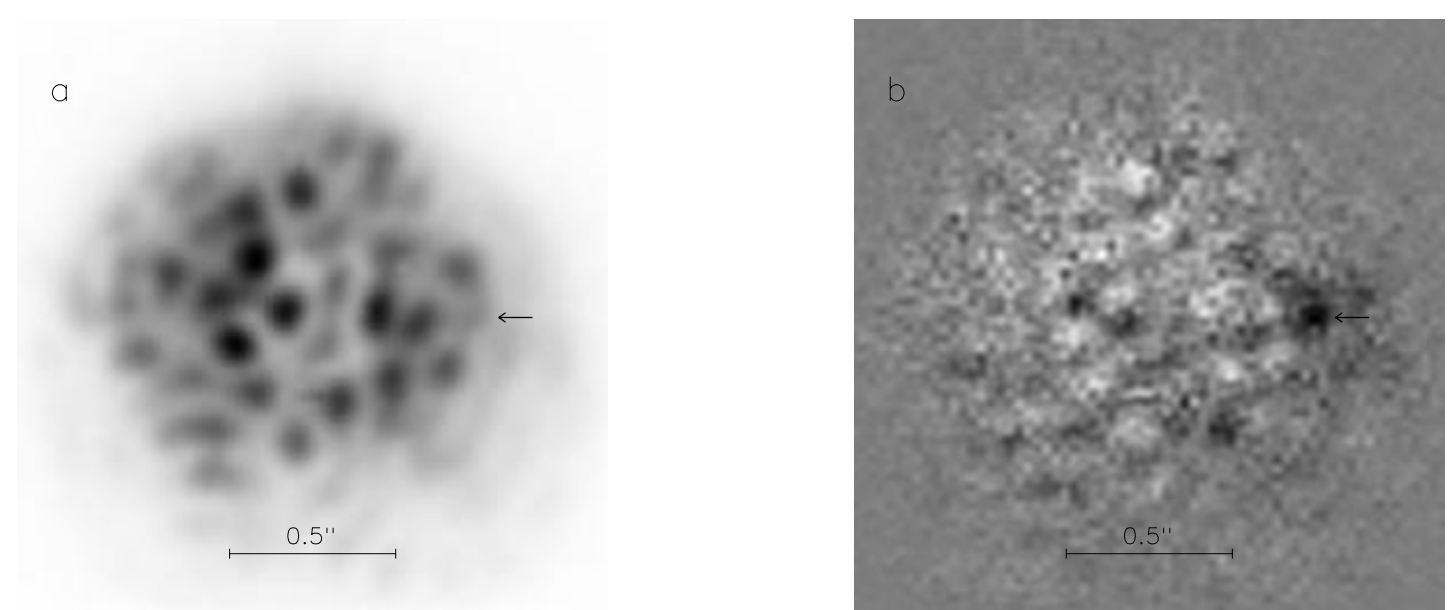

Fig. 3. Sample 2: Coronagraphic image of the binary a) and subtraction with a reference star b) for the sampling of $4 \times 4$ pixels/group, maximizing the $S N R$. The brightness ratio between the primary and the companion (arrow) is 7.3 magnitudes

Table 3. Parameters measured on long exposure, (sample 2)

\begin{tabular}{lccccc}
\hline & $\Delta m$ & $G$ & $S N R_{1}$ & $S N R \mathrm{th}_{1}$ & $S N R_{\mathrm{g}}$ \\
\hline $\begin{array}{l}\text { Long exposure } \\
1 \text { photo-event }\end{array}$ & 7.3 & 172 & 55.9 & 39.4 & 21.3 \\
\hline
\end{tabular}

Table 4. Dark-speckle $S N R$, (sample 2)

\begin{tabular}{lcccc}
\hline Sampling & $S N R_{\mathrm{ds}}$ & $S N R \mathrm{th}_{\mathrm{ds}}$ & $S N R \mathrm{sc}_{\mathrm{ds}}$ & $S N R_{\mathrm{g}}$ \\
\hline $1 \times 1$ pix/group & 3.1 & 16.5 & 37.3 & 20.7 \\
$2 \times 2$ pix/group & 16.8 & 31.6 & 104.7 & 38.0 \\
$4 \times 4$ pix/group & 62.2 & 54.1 & 196.5 & 48.5 \\
Theoritical & - & 71.9 & - & \\
\hline
\end{tabular}

when properly scaled. However, the dark-speckle imaging outperforms long exposure for pixel sampling of 38.3 and 9.5 pixels/speckle.

\section{Results}

For each data set we produce a coronagraphic image of the binary star and the subtraction process obtained with a sampling parameter of 9.5 pixels/speckle area $(4 \times 4$ pixels per group). In these samples, the optimal sampling is always smaller than the camera pixel sampling since the $R / G$ value is less than 10 and therefore maximal $S N R$ is actually achieved at 9.5 pixels/speckle area. However, for fainter companions $(R / G>>10)$ a finer sampling would be required,together with more short exposures.

For each sample, we also give 2 tables. The $1^{\text {st }}$ table presents the parameter infered from the long exposure, namely the brightness ratio, the AO gain $(G)$, and compares the measured $S N R\left(S N R_{\mathrm{l}}\right)$ with expected value from Eq. (4) $\left(S N R \mathrm{th}_{1}\right)$. The $2^{\text {nd }}$ table is then related to the dark-speckle process. The $1^{\text {st }}$ row gives the $S N R$ calculated on the negative "cleaned image", for each available samplings. A theoretical estimate is presented in the $2^{\text {nd }}$ row $\left(S N R \mathrm{th}_{\mathrm{ds}}\right)$. The $3^{\text {rd }}$ row gives the dark-speckle $S N R$ after scaling $\left(S N R \mathrm{sc}_{\mathrm{ds}}\right)$ for an accurate comparison of the long-exposure and dark-speckle images (Eq. (7)).

In addition, we have computed a more realistic signal to noise ratio including the global noise $\left(S N R_{\mathrm{g}}\right)$ on the subtracted images, namely the noise originating from the fixed speckle pattern. $S N R_{\mathrm{g}}$ has to be compared with $S N R_{1}$ and $S N R \mathrm{sc}_{\mathrm{ds}}$ respectively in tables related to the long exposure (Tables 1, 3, 5) and tables related to the dark-speckle exposure (Tables $2,4,6$ ). The $S N R$ obtained this way is considerably lower than other evaluated $S N R$ s.

The samples presented hereafter have been obtained under moderately good seeing conditions: $D / r_{0}=8.8$ at $0.65 \mu \mathrm{m}$ and $\bar{v}=0.39 \mathrm{~m} / \mathrm{s}$ for a $100 \mathrm{~mm}$ pupil $(\equiv 5.9 \mathrm{~m} / \mathrm{s}$ for a $1.5 \mathrm{~m}$ pupil). Depending on the room temperature regulation, $r_{0}$ ranges from $16 \mathrm{~cm}$ to $18 \mathrm{~cm}$ if scaled to a $1.5 \mathrm{~m}$ pupil.

In the following, we present the most relevant samples obtained in this laboratory simulation.

\subsection{Sample 1}

The first sample shows a companion 6.4 magnitudes fainter than the primary (Fig. 2). Binary star and reference star images were respectively reconstructed from 16143 and 6818 frames, each containing approximately 300 photon events. At this level, the brightest speckle can be saturated, but the companion, which is 2.4 times fainter than the average halo, is not affected. This saturation naturally decreases the halo/companion intensity ratio and may explain the discrepancy between measured and expected $S N R$ in long exposures (Table 1). The $S N R_{1}$ is indeed $63 \%$ higher than the expected value. No such behaviour was observed in sample 3 where the photon rate is lower than $170 \mathrm{ph} /$ frame. Also, since only the single photon events are exploited, the CP20 frames differ from a CCD long exposure which can 

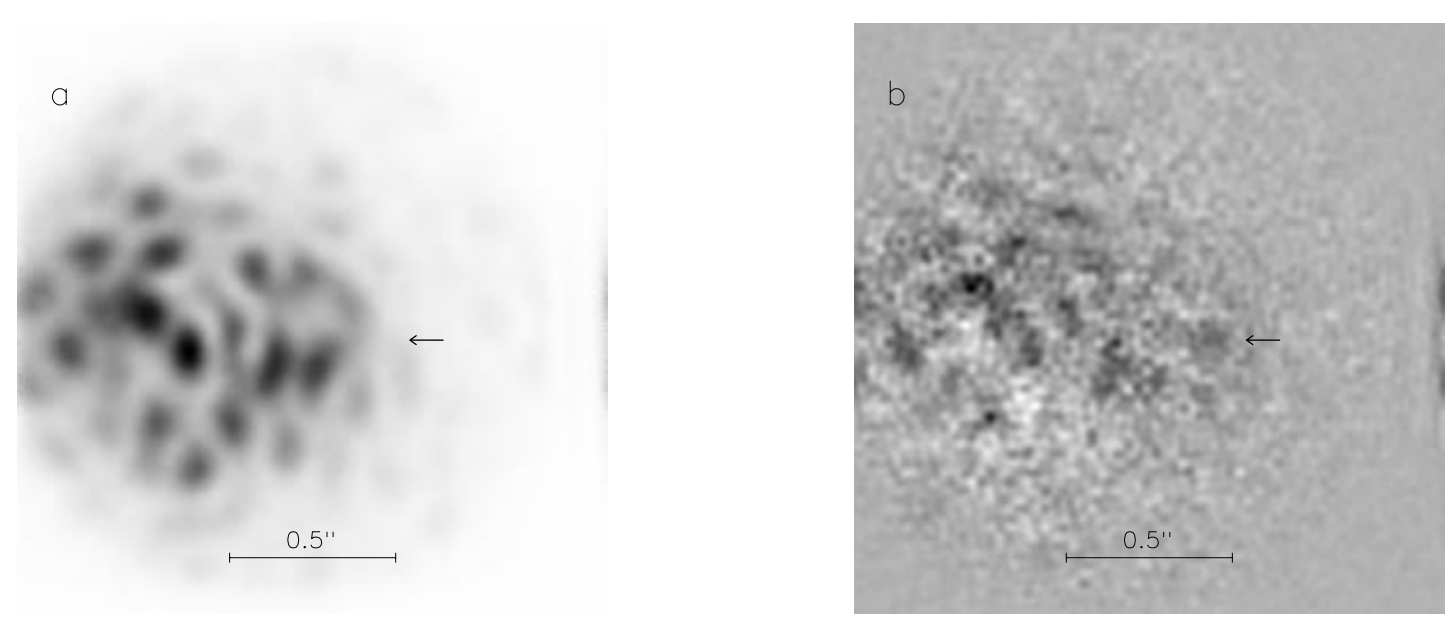

Fig. 4. Sample 3: Coronagraphic image of the binary a) and subtraction with a reference star b) for the sampling of $4 \times 4$ pixels/group, maximizing the $S N R$. The brightness ratio between the primary and the companion (arrow) is 7.8 magnitudes

Table 5. Parameters measured on long exposure, (sample 3)

\begin{tabular}{lccccc}
\hline & $\Delta m$ & $G$ & $S N R_{1}$ & $S N R \mathrm{th}_{1}$ & $S N R_{\mathrm{g}}$ \\
\hline $\begin{array}{l}\text { Long exposure } \\
1 \text { photo-event }\end{array}$ & 7.8 & 190 & 27.7 & 37.2 & 6.2 \\
\hline
\end{tabular}

Table 6. Dark-speckle $S N R$, (sample 3)

\begin{tabular}{lcccc}
\hline Sampling & $S N R_{\mathrm{ds}}$ & $S N R \mathrm{th}_{\mathrm{ds}}$ & $S N R \mathrm{sc}_{\mathrm{ds}}$ & $S N R_{\mathrm{g}}$ \\
\hline $1 \times 1$ pix/group & 1.6 & 7.7 & 27.7 & 6.2 \\
$2 \times 2$ pix/group & 4.6 & 15.0 & 37.9 & 9.5 \\
$4 \times 4$ pix/group & 18.0 & 27.5 & 76.2 & 8.5 \\
Theoritical & - & 36.7 & - & \\
\hline
\end{tabular}

contribute to this discrepancy. In addition, the measured and expected dark-speckle $S N R$ (Table 2) are consistent, at least for the $4 \times 4$ pixels binning $\left(S N R_{\mathrm{ds}}=107.3\right.$, $\left.S N R \mathrm{th}_{\mathrm{ds}}=97.4\right)$, and it suggests that the discrepancy mentionned hereabove, does not result from an overestimated intensity ratio. Indeed, dark-speckle images are not affected by the saturation.

Once the dark-speckle image is scaled to the long exposure, the $S N R$ obtained for both data with the finest sampling $(1 \times 1 \mathrm{pixel} /$ group $)$ are almost similar $\left(S N R_{1}=87.9\right.$, $\left.S N R \mathrm{sc}_{\mathrm{ds}}=78\right)$. Nevertheless, the best $S N R$ calculated in the dark-speckle image with $4 \times 4$ pixels/group, reaches 315 , which is considerably higher than for the long exposure. A more realistic value $\left(S N R_{\mathrm{g}}=80.8\right)$ is computed, including the global residual speckle noise in the coronagraphic image.

\subsection{Sample 2}

The sample 2 illustrates the detection of a fainter companion $(\Delta m=7.3)$ almost 5 times fainter than the average halo, after an integration of 38977 frames with a photon rate of $245 \mathrm{ph} /$ frame (Fig. 3). On the dark-speckle image (Table 4) with $1 \times 1 \mathrm{pixel} /$ group, the companion is almost undetectable and the measured $S N R\left(S N R_{\mathrm{ds}}=3.1\right)$

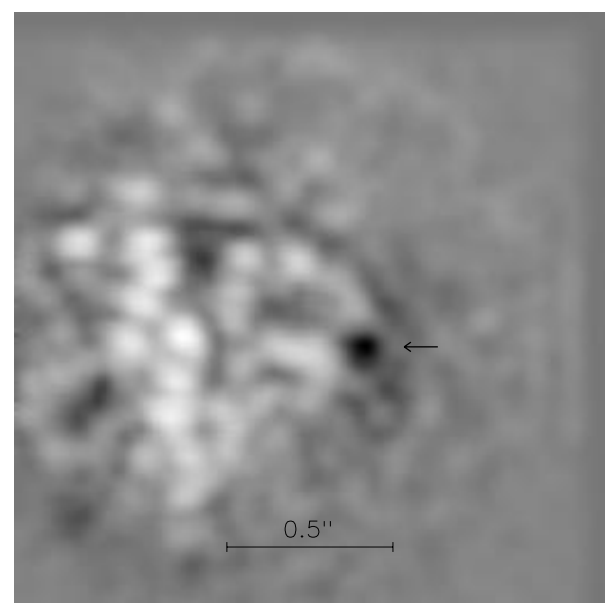

Fig. 5. $\chi^{2}$ map obtained with Fig. $4 \mathrm{~b}$ and a Gaussian shape to enhance the contrast of the companion. Companion's location is given by the minimum of $\chi^{2}$ (arrow)

is lower than its theoretical value $\left(S N R \mathrm{th}_{\mathrm{ds}}=16.5\right)$. After correct scaling of the dark-speckle image, the $S N R$ (37.3) becomes closer to one obtained in the long exposure (55.9). Measured dark-speckle $S N R$ approaches its expected value near the optimal sampling $\left(S N R_{\mathrm{ds}}=62.2\right.$, $\left.S N R \mathrm{th}_{\mathrm{ds}}=54.1\right)$. When compared accurately through proper scaling, the dark-speckle $S N R$ is then 3.5 times higher than the long-exposure $S N R$ (Table 4, third column). From the analysis of the global residual fluctuations in the image, we derive an $S N R_{\mathrm{g}}=48.5$ for the largest sampling.

\subsection{Sample 3}

This is the faintest companion detected in these laboratory tests $(\Delta m=7.8)$. A large number of frames (65856), representing 22 minutes of simulated observing, was required to obtain significant $S N R$ (Tables 5 and 6 ). Here again, the measured and theoretical dark-speckle $S N R$ are more 
similar for a sampling of 9.5 pixels/speckle area. On the subtracted image (Fig. 4) the companion's Airy peak is still not distinguishable from the brighter residual speckles in the coronagraphic field. Nevertheless, as explained in Sect. 3.2.3, to accurately assess the model, the $S N R$ was calculated in accordance with the local noise. We therefore derive an $S N R$ of about 28 for the finest sampling which is relatively high with respect to the residuals in Fig. 4. Taking into account the global residual speckle noise leads to more realistic values ranging between 6.2 and 9.5 depending on the pixel sampling.

The long exposure $S N R$ is close to the value expected from Eq. (4) $\left(S N R_{1}=27.7, S N R \mathrm{th}_{\mathrm{l}}=37.2\right)$. For $2 \times 2$ pixels grouping, we have measured an $S N R_{\mathrm{ds}}=4.6$ in the dark-speckle image, but a better agreement with theoretical value is achieved with $4 \times 4$ pixels /group $\left(S N R_{\mathrm{ds}}=18\right.$, $\left.S N R \mathrm{th}_{\mathrm{ds}}=27.5\right)$. Nevertheless, the scaled dark-speckle image shows that the $S N R$ is still higher $\left(S N R \mathrm{sc}_{\mathrm{ds}}=\right.$ 76.2 ). It demonstrates that fainter companions could have been detected in these tests.

To accurately distinguish the companion's Airy peak from brighter residual speckles, a $\chi^{2}$ test was carried out on the subtracted image of Fig. 4 . At each pixel a $\chi^{2}$ value is evaluated between the original data and a Gaussian shape, the width of which is constrained by the resolution. Fixed speckles are assumed differents in size and shape from a perfect Airy peak and are therefore efficiently removed with a $\chi^{2}$ test which has been found better than a simple correlation. The gaussian's amplitude is chosen to minimize the global minimum of the $\chi^{2}$ map indicating the location of the companion. Features such as companions close to a Gaussian shape and fainter that fixed speckles, are enhanced by the $\chi^{2}$ test. The Fig. 5 shows the result of this process applied on the subtracted image presented in Fig. 4. This refinement is obviously less effective if one of the bright speckle is superimposed to the companion's Airy peak. Also the $\chi^{2}$ test is restrained to point-like source.

\section{Summary of the laboratory tests}

These laboratory simulations were of interest to avoid uncontrollable disturbances arising in real observing variable seeing conditions, clouds, jitter, optical device alignment... Concluding remarks are as follows:

1: From a relatively good consistency of the long exposure $S N R$ with its theoretical expectation (Eq. 4), we have concluded that the AO gain and the intensity ratio have been accurately estimated;

2: The measured dark-speckle $S N R$ increases when the sampling approaches its optimal value (Eq. 1) and becomes closer to its theoretical expression (Eq. 5);

3: The scaled $S N R$ evaluated for the finest sampling $(1 \times 1 \mathrm{pixel} /$ group $)$ is similar to the long exposure $S N R$ owing to the duality between 0 and 1-photon events.
When the sampling is reduced, the scaled dark-speckle images clearly outperforms the long exposure;

4: The saturation of residual speckles may tend to reduce the contrast of the faint companion;

5: Present capabilities are limited by the residual static speckles. For accurate comparison with the dark-speckle model, we did not take into account these defects for the calculation of the $S N R$. In some conditions (see Sect. 4.3), a shape criterion can remove the brightest residual speckles which differ from a symmetrical Airy peak.

We have reported hereabove the detection of a companion 7 times fainter than the average halo (Sect. 4.3). Regarding the promising results obtained, the study suggests that even fainter companions could be imaged with the present instrument. The instrument sensitivity is derived from the results obtained in sample 3 . In the same integration time and seeing conditions, theoretical study based on dark-speckle analysis leads to a limited brightness ratio of about $\Delta m=10$. Taking the global speckle noise into account decreases this limit down to $\Delta m=$ 8. Nevertheless, it is expected that deeper imaging can be achieved with longer integration and higher photon rate. The detection of substellar companions is currently constrained by fixed patterns on coronagraphic images. Precise map subtraction, dark-speckle analysis and further refinements ( $\chi^{2}$ test for instance) are required for extracting the faintest levels around stars.

\section{Prospects and conclusion}

As discussed in Boccaletti et al. (1998b), the sensitivity of dark-speckle imaging is currently limited by the presence of a few fixed speckles in the cleaned image which results from small permanent residual phase errors on the wavefront, escaping correction by the adaptive optics. A local bias in the error signal or actuator response, causing the average phase value to be off-set from zero, generates such fixed speckles which survive the local intensity modulation in the multiple exposures. Subtraction of the cleaned image from a similar image obtained on a reference star improves the darkening, but removing most of the fixed speckles in the first place is highly desirable. In coronagraphic images these defects become dominant.

A possible way of reducing the effect of these fixed aberrations consists in boiling the static speckle pattern. This can be achieved in principle, if offset voltages on the adaptive mirror are randomly modulated around their average value with appropriate amplitude and higher frequencies, the stroke of the actuators being within the range of the $\mathrm{AO}$ servo noise. Although such a process smoothes the residual speckles and then increases the average halo intensity on the long exposure, it does not really affect the dark-speckle analysis which remains "not sensitive" to fluctuating speckles. 
The most critical device for the starlight cancellation is the coronagraph itself. New concepts in coronagraphy have been recently suggested: the achromatic interfero-coronagraph (AIC) (Gay \& Rabia 1996), and the phase-mask coronagraph (Rodier \& Roddier 1997). Both enhance faint companion imaging close to the Airy peak. The capability of such devices combined with a darkspeckle analysis remains to be thoroughly investigated.

As far as the detector is concerned the main present limitation is the saturation of the centroiding processor which implies low photon fluxes $(<300 \mathrm{ph} /$ frame). Higher photocathode efficiency and larger CCD chips, together with more powerful processors should improve the situation. Also important for high-contrast imaging is the abscence of internal scattered light within image intensifier tubes and CCDs. In addition, the feasibility of dark-speckle imaging with a near IR detector featuring a $38 \mathrm{e}^{-}$/pixel read-out noise is being studied.

Finally, these laboratory tests are considered a preliminary attempt in the context of NGST studies to design a concept of visible/nearIR and multipurpose coronagraphic capability (Gezari et al. 1997; Moutou et al. 1998; Rabbia et al. 1998; Lefevre et al. 1998). Exoplanets detection is presently a key goal of the design reference mission. Requirements and specifications for such an instrument are currently under evaluation (Labeyrie et al. 1999). In this context, intensive laboratory simulations will be required in the next few years and coronagraphic prototype should enable to assess the best solutions for future missions.

Acknowledgements. We are grateful to the ONERA team for their efficient support during this 15 days run. We especially thank B. Fleury (ONERA) for the control of the AO loop, A. Labeyrie (Observatoire de Haute-Provence) for helpful discussions and R. Burnage (Observatoire de Haute-Provence) for careful reading. We also congratulate V. Thevenet (Paris Observatory) for the precise realisation of the Lyot stops.

\section{References}

Abe L., Vakili F., Percheron I., Hamma S., Ragey J.P., Blazit A., 1998, Proceedings of the conference "Catching the Perfect Wave", Albuquerque N.M. (eds.), (June 1998)

Angel J.R.P., 1994, Nat 368, 203

Boccaletti A., Labeyrie A., Ragazzoni R., 1998, A\&A 338, 106 Boccaletti A., Moutou C., Labeyrie A., Kohler D., Vakili F., 1998, A\&AS 133, 395

Conan J.-M., Mugnier L., Fusco T., 1998, SPIE 3126, Proceedings of the Adaptive Optics and Applications

Gay J., Rabbia Y., 1996, CR. Acad. Sci. Paris, t. 332, Serie II b, p. 265

Gezari D.Y., Crotts A., Danchi W., et al., 1997, Columbia University and Ball Aerospace Systems Division, proposal in response to NRA 98-GSFC-1

Labeyrie A., 1995, A\&A 298, 544

Labeyrie A., Vakili F., Abe L., Moutou C., Boccaletti A., Schneider J., et al., 1999 (in preparation)

Le Fèvre O., et al., 1998, Proposal to ESA (Dornier, LAS)

Lyot B., 1939, MNRAS 99, 580

Malbet F., Yu J.W., Shao M., 1995, PASP 107, 386

Malbet F., 1996, A\&AS 115, 161

Moutou C., Boccaletti A., Labeyrie A., 1998, Proceedings of the $34^{\mathrm{e}}$ Liège Astrophysics Colloquium, The NGST: Science Drivers and Technological Challenges, Liège, 1998, p. 211

Rabbia Y., Baudoz P., Gay J., 1998, Proceedings of the $34^{\mathrm{e}}$ Liège Astrophysics Colloquium, The NGST: Science Drivers and Technological Challenges, Liège, 1998, p. 279

Roddier F., Roddier C., 1997, PASP 109, 815

Thiébaut E., 1994, A\&A 284, 340

Wynne C.G., 1979, Opt. Comm. 28, 21 